\title{
Black hole solutions in the warped DGP braneworld
}

\author{
Kourosh Nozari $^{*}$ and Amir Asaiyan ${ }^{\dagger}$ \\ Department of Physics, \\ Faculty of Basic Sciences, \\ University of Mazandaran, \\ P. O. Box 47416-95447, Babolsar, IRAN
}

\begin{abstract}
We study the static, analytical solution of black holes in the warped DGP braneworld scenario. We show that the linearized field equations and matching conditions lead to solutions that are not compatible with Schwarzschild- $(\mathrm{A}) \mathrm{dS}_{(4)}$ solutions on the brane. This incompatibility is similar to vDVZ discontinuity in massive gravity theory. Following the standard procedure to remove this discontinuity, which firstly was proposed by Vainshtein, we keep some appropriate nonlinear terms in the field equations. This strategy has its origin in the fact that the spatial extrinsic curvature of the brane plays a crucial role in the nonlinear nature of the solutions and also in recovering the wellmeasured predictions of General Relativity (GR) at small scales. Using this feature, we obtained an interesting black string solution in the bulk when it is compatible with 4D GR solutions on the brane.
\end{abstract}

PACS: 04.70.-s, 04.50.-h

Key Words: Black Holes, Braneworlds, vDVZ Discontinuity.

*knozari@umz.ac.ir

†a.asaiyan@umz.ac.ir 


\section{Introduction}

A model universe with possible extra dimensions has attracted a lot of attention in recent years. Questions such as: are we living really in a four dimensional universe, or are there extra dimensions we cannot see, have not been addressed precisely yet. But, theoretically there are a lot of interesting outcomes by incorporating some extra dimensions in theories such as gravitation. In fact, extra dimensions are an integral part of fundamental theories of physics such as superstring/M-theories that need more than four spacetime dimensions. The idea to add an extra dimension to General Relativity's four dimensional spacetime, firstly was introduced by Kaluza and Klein to unify Electromagnetic and Gravity. Now p-branes and $D$-branes are well-known in the context of string theory where branes are solitonic solutions of 10-dimensional string theories. $p$-branes are extended objects of higher dimension than strings (1-branes) and the $D$-branes are kind of $p$-branes on which open strings can end [1]. In braneworld scenarios we assume that our $(1+3)$-dimensional spacetime is a domain wall embedded in a 5-dimensional spacetime called the bulk [1]. All matter fields and other gauge bosons live on the brane but gravitons, which carry the gravitational interaction, can travel into the extra dimension. There are at least two basic reasons for investigating braneworld models: firstly for solving hierarchy problem, that is, a large difference between Planck and Electroweak scales, secondly for proposing a unified theory of all fundamental interactions in the nature.

During the last two decades, different kinds of braneworld scenarios have been proposed such as the Horava-Witten model [2], Arkani Hamed-Dimopoulos-Dvali (ADD) model [3], Randall-Sundrum (RS) models [4] and the Dvali-Gabadadze-Porrati (DGP) model [5]. Among these models, one of which has attracted most regards in last decade, is the RS one brane (RSII) model. In this model it is assumed that our universe is a 3-brane with positive tension, the extra dimension is large and the bulk has a negative cosmological constant leading to a warped geometry. Another model that has attractive results from cosmological viewpoint, is the DGP model with one large extra dimension but the bulk is Minkowski. In this model, there is a new term in the total action that comes from quantum interaction between matter confined on the brane and the bulk gravitons which induces gravity on the brane. In this model, gravity leaks off the 4D brane into the bulk and becomes 5-dimensional at large scales, $r \gg r_{c}$, but on small scales, gravity is effectively bounded to the brane and 4D dynamics is regained. The transition from $4 \mathrm{D}$ to $5 \mathrm{D}$ behavior is governed by the crossover scale $r_{c}$. An interesting property of this model is that it contains a self-accelerating branch of the solutions which can explain late time acceleration due to a weakening of gravity at low energies [8].

We note that a basic requirement for any alternative theory of gravity, such as braneworld scenarios, is that they should reproduce General Relativity predictions in the appropriate limit to be phenomenologically viable. Two of the most important applications of GR are cosmology and black holes. Here, our concentration is on the issue of braneworld black holes, i.e. finding the bulk and the brane metric when a spherically symmetric energy-momentum distribution is localized on the brane. To obtain black hole solutions in a braneworld scenario, we note that generally braneworld solutions can be obtained by following two different approaches: In the first approach, dynamics and geometry of the whole bulk spacetime are primarily considered, then the dynamics on the brane is extracted by using the DarmoisIsrael matching conditions. The second approach is to obtain effective 4-dimensional field equations on the brane firstly and then try to extent these solutions to the bulk. This approach has been applied to braneworlds with induced gravity in Ref. [6]. The main difficulty 
in the later approach is that it is not always possible to obtain a closed set of equations on the brane. So, we are not able to predict the behavior of the fields on the brane just with data on the brane.

In 1999, Chamblin, Hawking and Reall found the Schwarzschild black string solution for RSII model as follows [10]

$$
\begin{gathered}
d s_{5}{ }^{2}=e^{\frac{-2|y|}{\ell}} \tilde{g}_{\mu \nu} d x^{\mu} d x^{\nu}+d y^{2} \\
\tilde{g}_{\mu \nu}=e^{\frac{2|y|}{\ell}} g_{\mu \nu}=-\left(1-\frac{2 G M}{r}\right) d t^{2}+\frac{d r^{2}}{1-\frac{2 G M}{r}}+r^{2} d \Omega^{2} .
\end{gathered}
$$

In this solution, there is a line singularity along $r=0$ for all $y$, but the black string is unstable in response to large-scale perturbations because the 5D curvature is unbounded at the Cauchy horizon, as $y \rightarrow \infty$. Another solution for static uncharged black hole was found by Dadhich et al. from the induced field equations on the brane [11]. In this work, a Reissner-Nordström type correction to the Schwarzschild solution was found and 5D gravitational effects, which are impressed by the bulk Weyl tensor, induce a tidal charge parameter $Q$. In this approach the bulk metric has not been found. Braneworld black holes have been studied extensively in recent years, some of these studies can be found in Refs. [10]-[17]. An extension of the DGP setup is the warped DGP scenario, which is a hybrid braneworld model containing both RSII and the DGP models as its limits $[6,7]$. This model is constructed by considering the effect of an induced gravity term in the RSII model. Black hole solutions in a warped DGP braneworld have been studied recently in the case of a conformally flat bulk for spherically symmetric vacuum on the brane [18]. This study confirms the idea that an extra term in the effective vacuum field equations on the brane can play the role of a positive cosmological constant.

Within this streamline, in this paper we present an analytical solution of static black hole in the warped DGP braneworld. Firstly, we consider pure $(A) d S_{(5)}$ bulk field equations with a general spherically symmetric 5D metric to achieve independent field equations that should be held in the bulk. We take into account the 3-brane effect through appropriate matching conditions that act as boundary conditions on 3-brane universe in order to constraint general bulk solutions. In this way, we obtain a complicate system of equations which should be solved to reach the warped DGP braneworld, static, black hole solutions. Due to difficulties in solving the field equations analytically, we consider the weak field limit of the scenario to find those solutions of the field equations that describe the gravitational interactions in regions far enough from the gravitational source. We demand these solutions to support 4D General Relativity solutions for $r \ll r_{c}$ on the brane. However, the full linearized theory that we have adopted, does not lead to a correct 4D Schwarzschild-(A)dS $\mathrm{S}_{(4)}$ solution on the brane. The reason for this incompatibility and its solution are summarized in which follows: The linear analysis of the DGP model shows that the tensor structure of the induced metric perturbations takes the five dimensional form even at short distances, i.e. in the limit $r_{c} \rightarrow \infty$, we have an incompatibility with Einstein gravity $[5,8,9]$. The situation is analogous to the case of models with massive gravitons in which we have a deviation from 4D GR (which is a massless theory), even in the massless limit. This discontinuity in graviton mass is known as van Dam-Veltman-Zakharov (vDVZ) discontinuity [19]. Vainshtein claimed that the vDVZ discontinuity is an artifact of the Pauli-Fierz Lagrangian, i.e. of the linearization of the true, covariant, non-linear equations of massive gravity, and the $4 \mathrm{D}$ GR is recovered by non-linear effect [20]. There have been many discussions about this issue, see for instance [21]. Another possibility that can disappear the mentioned discontinuity is introducing a cosmological constant [22]. In this way, in order to 4D GR be recovered, the cosmological 
constant should be large enough as far as the graviton mass is not completely negligibly small. The paradox in DGP gravity seems to be that while it is clear that a perturbative, vDVZ-like discontinuity occurs in the potential somewhere, no such discontinuity appears in the cosmological solutions; at large Hubble scales, the theory on the brane appears safely like general relativity $[8,9]$. Indeed, the cosmological solutions at large Hubble scales are extremely nonlinear, and that perhaps, just as Vainshtein suggested for massive gravity, nonlinear effects become important in resolving the DGP version of the vDVZ discontinuity. In the context of DGP model, there are various works that indicate $4 \mathrm{D}$ GR is recovered at short distances $[23,24]$. It was shown that the brane bending becomes non-linear at scale shorter than $r_{*} \equiv\left(r_{c}^{2} r_{g}\right)^{1 / 3}$, thus the linear analysis breaks down there. Also the leading order correction to 4D GR has been obtained in this regime [24]. In Ref. [25], an alternative formalism which can handle general perturbations in weak gravity regime was proposed. They generalized the DGP model by taking into account the bulk cosmological constant and the brane tension balanced with it, so the background metric is $\operatorname{AdS}_{(5)}$ in the bulk and Minkowski on the brane. By perturbing the background metric and following the prior approach that had been proposed for RS scenario [26], they confirmed the recovery of 4D GR at short distances and re-derived the leading order correction to it. They have done this work by taking into account the non-linear brane bending for weak gravity at small scale $r<r_{*}$.

Here, in order to recover 4D Schwarzschild-(A)dS $\mathrm{dS}_{(4)}$ solution on the brane for $r<r_{*}$ or in the limit $r_{c} \rightarrow \infty$, with regard to previous works, we take into account some nonlinear terms in the field equations. In fact, these nonlinear terms become so large in that regions that one cannot neglect them relative to other nonlinear terms. We show that nonlinear effects become important in resolving the "Warped DGP" version of the vDVZ discontinuity too. By considering these nonlinear terms, we have found interesting solutions for static Black holes on the warped DGP brane.

\section{The field equations}

We consider a 3-brane $\Sigma$ embedded in a 5 -dimensional bulk $\mathcal{M}$. The total action for the system is

$$
S=\frac{1}{2 \kappa_{5}^{2}} \int_{\mathcal{M}} d^{5} X \sqrt{-g}\left({ }^{(5)} R-2 \Lambda_{5}\right)+\int_{\mathcal{M}} d^{5} X \sqrt{-g} \mathcal{L}_{\text {bulk }}+S_{\text {brane }}
$$

where $S_{\text {brane }}$ is the 3 -brane action defined as

$$
S_{\text {brane }}=\frac{1}{2 \kappa_{4}^{2}} \int_{\Sigma} d^{4} x \sqrt{-q}\left({ }^{(4)} R-2 \Lambda_{4}\right)+\int_{\Sigma} d^{4} x \sqrt{-q} \mathcal{L}_{\text {brane }}+\int_{\Sigma} d^{4} x \sqrt{-q} \frac{K}{\kappa_{5}^{2}} .
$$

$g_{A B}$ is the $5 \mathrm{D}$ bulk metric with corresponding Ricci tensor given by ${ }^{(5)} R_{A B}$. The brane has induced metric $q_{\mu \nu}$ with corresponding Ricci tensor ${ }^{(4)} R_{\mu \nu}$. The boundary Gibbons-Hawking term is implied to yield the correct Einstein equations in the bulk. $\Lambda_{5}$ and $\Lambda_{4}$ are bulk

and brane cosmological constants respectively. $\frac{\Lambda_{4}}{\kappa_{4}^{2}}$ can be interpreted as the brane tension of the standard Dirac-Nambu-Goto action and can include quantum contributions to the four-dimensional cosmological constant [12]. $\mathcal{L}_{\text {bulk }}$ and $\mathcal{L}_{\text {brane }}$ are the bulk and brane matter Lagrangian respectively. We note also that the Planck masses $M_{5}$ and $M_{4}$ are defined as 
$\kappa_{5}^{2}=8 \pi G_{(5)}=M_{5}^{-3}$ and $\kappa_{4}^{2}=8 \pi G_{(4)}=M_{4}^{-2}$, where $M_{5}$ and $M_{4}$ have dimensions of $(\text { length })^{-1}$.

We chose a coordinate $y$ for extra dimension so that our 3-brane in the Gaussian Normal Coordinates is localized at $y=0$. By variation of the action with respect to the bulk metric, $5 \mathrm{D}$ field equations would be obtained as follows

$$
{ }^{(5)} G_{A B}=-\Lambda_{5} g_{A B}+\kappa_{5}^{2(b u l k)} T_{A B}+\delta(y) \kappa_{5}^{2}{ }^{(l o c)} T_{A B}
$$

where

$$
{ }^{\left({ }^{(o c)}\right)} T_{A B}=g_{A}^{\mu} g_{B}^{\nu}\left(-\frac{1}{\kappa_{4}^{2}}\right) \sqrt{\frac{-q}{-g}}\left({ }^{(4)} G_{\mu \nu}+\Lambda_{4} q_{\mu \nu}-\kappa_{4}^{2{ }^{(\text {brane })}} T_{\mu \nu}\right),
$$

is the localized energy-momentum tensor on the brane and

$$
{ }^{\left({ }^{(b u l k)}\right.} T_{A B}=-2 \frac{\delta \mathcal{L}_{\text {bulk }}}{\delta g^{A B}}+g_{A B} \mathcal{L}_{\text {bulk }}
$$

${ }^{(5)} G_{A B}$ and ${ }^{(4)} G_{\mu \nu}$ denote the Einstein tensors constructed from the bulk and the brane metrics respectively. The tensor $q_{\mu \nu}$ is the induced metric on the brane $\Sigma$. The field equations in the bulk take the following form

$$
{ }^{(5)} G_{A B}={ }^{(5)} R_{A B}-\frac{1}{2}{ }^{(5)} R g_{A B}=-\Lambda_{5} g_{A B}+\kappa_{5}^{2}{ }^{(b u l k)} T_{A B},
$$

or

$$
{ }^{(5)} R_{A}^{B}-\frac{1}{2}{ }^{(5)} R \delta_{A}^{B}=-\Lambda_{5} \delta_{A}^{B}+\kappa_{5}^{2}{ }^{(b u l k)} T_{A}^{B} .
$$

Moreover, the following modified (due to the presence of induced gravity on the brane) Israel-Darmois junction conditions would be obtained from the distributional character of the brane content

$$
\left[K_{\mu \nu}\right]-q_{\mu \nu}[K]=-\kappa_{5}^{2}{ }^{(l o c)} T_{\mu \nu}=\frac{\kappa_{5}^{2}}{\kappa_{4}^{2}}\left({ }^{(4)} G_{\mu \nu}+\Lambda_{4} q_{\mu \nu}\right)-\kappa_{5}^{2}{ }^{(\text {brane })} T_{\mu \nu}
$$

where $K_{\mu \nu}=\frac{1}{2} \partial_{y}\left(g_{\mu \nu}\right)$ is the extrinsic curvature of the brane and brackets denote jump across the brane $(y=0)$.

Now we consider a general, 5-dimensional, static metric with spherical symmetry on the brane as follows

$$
d s_{5}{ }^{2}=-e^{\nu(r, y)} d t^{2}+e^{\lambda(r, y)} d r^{2}+r^{2} e^{\mu(r, y)}\left(d \theta^{2}+\sin ^{2} \theta d \phi^{2}\right)+d y^{2} .
$$

Also we assume the matter content of the bulk is just a cosmological constant $\Lambda_{5}$ (i.e., $\left.{ }^{\left({ }^{(b u l k)}\right.} T_{A B}=0\right)$ and the matter content of the 3-brane universe is considered to be a cosmological constant $\Lambda_{4}$ plus a localized spherically symmetric untilted perfect fluid (e.g. a star) with

$$
{ }^{(\text {brane })} T_{\mu \nu}=(\rho+p) u_{\mu} u_{\nu}+p q_{\mu \nu},
$$

where $u^{\mu}$ stands for the 4-velocity of the fluid and $\rho=p=0$ for $r>R$. Nevertheless, since we want to obtain static black hole solutions outside the star ( that is, for $r>R$ ), in these regions the brane has just a cosmological constant $\Lambda_{4}$. 
Hereafter, we consider a $\mathbf{Z}_{2}$-symmetry on reflection across the brane, thus at $r>R$ the Israel matching conditions become

$$
\bar{K}_{\mu}^{\nu}-\bar{K} \delta_{\mu}^{\nu}=r_{c}\left({ }^{(4)} G_{\mu}^{\nu}+\Lambda_{4} \delta_{\mu}^{\nu}\right)
$$

where $r_{c}=\frac{\kappa_{5}^{2}}{2 \kappa_{4}^{2}}=\frac{M_{4}^{2}}{2 M_{5}^{3}}$ is the DGP crossover distance, and by definition $\bar{K}_{\mu}^{\nu}=K_{\mu}^{\nu}\left(y=0^{+}\right)=$ $-K_{\mu}^{\nu}\left(y=0^{-}\right)$. Now by putting the metric (9) into the bulk field equations (7) we obtain the $(t t),(r r),(\theta \theta)$ and $(r y)$ components of the bulk field equations respectively as follows

$$
\begin{gathered}
4 r \mu_{r r}+3 r \mu_{r}^{2}+12 \mu_{r}-4 \lambda_{r}-2 r \lambda_{r} \mu_{r}+\frac{4}{r}\left(1-e^{\lambda-\mu}\right)+ \\
2 r \lambda_{y y} e^{\lambda}+4 r \mu_{y y} e^{\lambda}+r \lambda_{y}^{2} e^{\lambda}+3 r \mu_{y}^{2} e^{\lambda}+2 r \lambda_{y} \mu_{y} e^{\lambda}=4 r \Lambda_{5} e^{\lambda}, \\
r \mu_{r}^{2}+2 r \nu_{r} \mu_{r}+4 \nu_{r}+4 \mu_{r}+\frac{4}{r}\left(1-e^{\lambda-\mu}\right)+2 r \nu_{y y} e^{\lambda}+ \\
4 r \mu_{y y} e^{\lambda}+r \nu_{y}^{2} e^{\lambda}+3 r \mu_{y}^{2} e^{\lambda}+2 r \nu_{y} \mu_{y} e^{\lambda}=4 r \Lambda_{5} e^{\lambda}, \\
2 r\left[\nu_{y y}+\lambda_{y y}+\mu_{y y}\right] e^{\lambda}+r\left[\lambda_{y} \nu_{y}+\lambda_{y} \mu_{y}+\nu_{y} \mu_{y}\right] e^{\lambda}+r\left[\lambda_{y}^{2}+\nu_{y}^{2}+\mu_{y}^{2}\right] e^{\lambda}=4 r \Lambda_{5} e^{\lambda}, \\
4 r \mu_{r r}+2 r \nu_{r r}+3 r \mu_{r}^{2}+r \nu_{r}^{2}+2 r \nu_{r} \mu_{r}-2 r \lambda_{r} \mu_{r}-r \nu_{r} \lambda_{r}+4 \nu_{r}-4 \lambda_{r}+12 \mu_{r}+ \\
\frac{4}{r}\left(1-e^{\lambda-\mu}\right)+r \mu_{y}^{2} e^{\lambda}+r\left[\lambda_{y} \nu_{y}+2 \lambda_{y} \mu_{y}+4 \nu_{y} \mu_{y}\right] e^{\lambda}=4 r \Lambda_{5} e^{\lambda}, \\
2 \nu_{r y}+4 \mu_{r y}+\nu_{r} \nu_{y}-\nu_{r} \lambda_{y}+2 \mu_{r} \mu_{y}-2 \lambda_{y} \mu_{r}+\frac{4}{r}\left(\mu_{y}-\lambda_{y}\right)=0 .
\end{gathered}
$$
brane

By using the Israel matching conditions (11), we obtain the following conditions on the

$$
\begin{gathered}
-\left.\frac{1}{2}\left(2 \mu_{y}+\lambda_{y}\right)\right|_{y=0^{+}}=r_{c}\left\{\Lambda_{4}-\frac{e^{-\lambda}}{4 r^{2}}\left(4-4 e^{\lambda-\mu}+4 r^{2} \mu_{r r}+3 r^{2} \mu_{r}^{2}-4 r \lambda_{r}+12 r \mu_{r}-2 r^{2} \lambda_{r} \mu_{r}\right)\right\}, \\
-\left.\frac{1}{2}\left(2 \mu_{y}+\nu_{y}\right)\right|_{y=0^{+}}=r_{c}\left\{\Lambda_{4}-\frac{e^{-\lambda}}{4 r^{2}}\left(4-4 e^{\lambda-\mu}+r^{2} \mu_{r}^{2}+4 r \mu_{r}+4 r \nu_{r}+2 r^{2} \nu_{r} \mu_{r}\right)\right\}, \\
-\left.\frac{1}{2}\left(\nu_{y}+\lambda_{y}+\mu_{y}\right)\right|_{y=0^{+}}=r_{c}\left\{\Lambda_{4}-\frac{e^{-\lambda}}{4 r}\left(2 r \nu_{r r}+2 r \mu_{r r}+r \mu_{r}^{2}+r \nu_{r}^{2}\right.\right. \\
\left.\left.-r \nu_{r} \lambda_{r}+2 \nu_{r}-2 \lambda_{r}+4 \mu_{r}+r \nu_{r} \mu_{r}-r \lambda_{r} \mu_{r}\right)\right\} .
\end{gathered}
$$

The subscripts $y$ and $r$ in these relations represent partial differentiation with respect to $y$ and $r$ respectively. Note that these equations are held on the brane outside our spherical object. 


\section{The weak field solutions}

The nonlinear, second order partial differential equations (12) - (16) should be solved and the corresponding solutions should satisfy junction conditions (17), (18) and (19) across the $\mathbf{Z}_{2}$-symmetric brane. This is not an easy task at all! To find some analytical solutions, we consider just the weak field regime (i.e., far enough from the source localized on the brane). In this respect, we adopt the assumption that $|\nu|,|\lambda|$ and $|\mu|$ are small quantities compared to unity; that is, $|\nu|,|\lambda|,|\mu| \ll 1$. By adopting this assumption, we linearize our field equations and also brane boundary conditions. By keeping only the leading order terms, the bulk equations (12) - (16) reduce to the following equations

$$
\begin{gathered}
2 r \mu_{r r}+4 \mu_{r}-2 \lambda_{r}-\nu_{r}-\frac{c}{r^{2}}+r\left(\lambda_{y y}+2 \mu_{y y}\right)=\frac{4}{3} r \Lambda_{5}, \\
\nu_{r}-\frac{c}{r^{2}}+r\left(\nu_{y y}+2 \mu_{y y}\right)=\frac{4}{3} r \Lambda_{5}, \\
r\left(\nu_{r r}+\mu_{r r}\right)+\nu_{r}+2 \mu_{r}-\lambda_{r}+r\left(\nu_{y y}+\lambda_{y y}+\mu_{y y}\right)=2 r \Lambda_{5}, \\
\frac{2}{r}(\lambda-\mu)=\nu_{r}+2 \mu_{r}+\frac{c}{r^{2}}-\frac{2}{3} r \Lambda_{5},
\end{gathered}
$$

where $c$ is an integration constant to be fixed later. In the same procedure applied to equations (17), (18) and (19), we achieve the following linearized junction conditions

$$
\begin{gathered}
-\left.\frac{1}{2}\left(\lambda_{y}+2 \mu_{y}\right)\right|_{y=0^{+}}=r_{c}\left\{\Lambda_{4}-\frac{1}{r^{2}}\left(\mu-\lambda+3 r \mu_{r}+r^{2} \mu_{r r}-r \lambda_{r}\right)\right\}, \\
-\left.\frac{1}{2}\left(\nu_{y}+2 \mu_{y}\right)\right|_{y=0^{+}}=r_{c}\left\{\Lambda_{4}-\frac{1}{r^{2}}\left(\mu-\lambda+r \mu_{r}+r \nu_{r}\right)\right\}, \\
-\left.\frac{1}{2}\left(\nu_{y}+\lambda_{y}+\mu_{y}\right)\right|_{y=0^{+}}=r_{c}\left\{\Lambda_{4}-\frac{1}{2 r}\left(r \nu_{r r}+r \mu_{r r}+2 \mu_{r}+\nu_{r}-\lambda_{r}\right)\right\} .
\end{gathered}
$$

By solving the bulk field equations (20) - (23), we find $\mu$ and $\lambda$ in terms of $\nu$ as follows

$$
\begin{gathered}
\mu(r, y)=-\frac{1}{2 r} \iint \nu_{r} d y d y-\frac{1}{2} \nu+\frac{c}{4 r^{3}} y^{2}+\frac{1}{3} \Lambda_{5} y^{2}+\left(F_{1}(r)+H(r)\right)|y|+F_{2}(r)+G(r), \\
\lambda(r, y)=-2 \iint \nu_{r r} d y d y-\frac{3}{r} \iint \nu_{r} d y d y-2 \nu+\frac{c}{2 r}+\frac{2}{3} \Lambda_{5} r^{2}+r|y| F_{1}^{\prime}(r)+ \\
|y| F_{1}(r)+r F_{2}^{\prime}(r)+F_{2}(r)+\frac{4}{3} \Lambda_{5} y^{2}-\frac{c}{2 r^{3}} y^{2}+A(r)|y|+B(r),
\end{gathered}
$$

where $A(r), B(r), F_{1}(r), F_{2}(r), G(r)$ and $H(r)$ are arbitrary functions of $r$, and a prime denotes derivative with respect to $r$. Also $\nu(r, y)$ should satisfy the following differential equation

$$
\nu_{r r y y}=-\nu_{y y y y}-\frac{2}{r^{3}} \nu_{r}-\frac{1}{r} \nu_{r y y}+\frac{1}{r} \nu_{r r r}+\frac{2}{r^{2}} \nu_{r r} .
$$


Therefore, for a given $\nu(r, y)$ that satisfies this equation, $\mu(r, y)$ and $\lambda(r, y)$ can be obtained via (27) and (28) respectively. Equation (29) has the following solution for $\nu(r, y)$

$$
\nu(r, y)=\frac{a}{r}+b r^{2}+e y^{2}+d|y|+\frac{f}{r}|y|+q r^{2}|y| .
$$

Now by using equations (27) and (28), we calculate $\lambda(r, y)$ and $\mu(r, y)$ to find

$$
\begin{gathered}
\lambda(r, y)=\left(-\frac{f}{6 r^{3}}-\frac{5}{3} q\right)|y|^{3}-\left(\frac{(a+c)}{2 r^{3}}+\frac{b}{2}+2 e-\frac{4}{3} \Lambda_{5}\right) y^{2}- \\
\left(2 q r^{2}+\frac{2}{r} f+2 d-r F_{1}^{\prime}-F_{1}-P(r)\right)|y|+\frac{c-4 a}{2 r}-2 b r^{2}+\frac{2}{3} \Lambda_{5} r^{2}+r F_{2}^{\prime}+F_{2}+Q(r), \\
\mu(r, y)=\left(\frac{f}{12 r^{3}}-\frac{q}{6}\right)|y|^{3}+\left(\frac{(a+c)}{4 r^{3}}+\frac{1}{3} \Lambda_{5}+\frac{b}{4}-\frac{e}{2}\right) y^{2}- \\
\left(\frac{f}{2 r}+\frac{q}{2} r^{2}+\frac{d}{2}-F_{1}-M(r)\right)|y|-\frac{a}{2 r}-\frac{b}{2} r^{2}+F_{2}+N(r),
\end{gathered}
$$

where $a, b, d, e, f$ and $q$ are constants, whereas $P(r), Q(r), M(r)$ and $N(r)$ are arbitrary functions of $r$. Equations (20) - (26) can help us to determine the arbitrary parameters and functions appeared in $\nu(r, y), \lambda(r, y)$ and $\mu(r, y)$. By this way, we found that $b, d, f$ and $q$ should be zero and also $\Lambda_{5}=-\Lambda_{4}$ and $e=\frac{2}{3} \Lambda_{5}$ in order for equations (20) - (26) to be satisfied. In this situation the solutions simplify to the following equations

$$
\begin{gathered}
\nu(r, y)=\frac{a}{r}+\frac{2}{3} \Lambda_{5} y^{2} \\
\lambda(r, y)=-\left(\frac{a+c}{2 r^{3}}\right) y^{2}+r_{c}\left(\frac{4}{3} \Lambda_{5}+\frac{a+c}{r^{3}}\right)|y|+\frac{c-a}{2 r}-\frac{1}{3} \Lambda_{5} r^{2}+r W^{\prime}+W(r), \\
\mu(r, y)=\left(\frac{a+c}{4 r^{3}}\right) y^{2}+r_{c}\left(\frac{4}{3} \Lambda_{5}-\frac{a+c}{2 r^{3}}\right)|y|+W(r)-\frac{a}{2 r},
\end{gathered}
$$

where $W(r)=N(r)+F_{2}(r)$. The corresponding quantities on the brane $(y=0)$ take the following forms

$$
\begin{gathered}
\nu(r, y=0)=\frac{a}{r}, \\
\lambda(r, y=0)=\frac{c-a}{2 r}+\frac{1}{3} \Lambda_{4} r^{2}+r W^{\prime}+W(r), \\
\mu(r, y=0)=W(r)-\frac{a}{2 r} .
\end{gathered}
$$

Since we expect $\mu(r, y=0)=0$, we set $W(r)=\frac{a}{2 r}$. So we find

$$
\lambda(r, y=0)=\frac{c-a}{2 r}+\frac{1}{3} \Lambda_{4} r^{2} .
$$

$c$ is an integration constant which we set to be zero in order to find more familiar results as follows 


$$
\begin{gathered}
\nu(r, y)=\frac{a}{r}+\frac{2}{3} \Lambda_{5} y^{2} \\
\lambda(r, y)=-\frac{a}{2 r}+\frac{1}{3} \Lambda_{4} r^{2}-\frac{a}{2 r^{3}} y^{2}+r_{c}\left(\frac{4}{3} \Lambda_{5}+\frac{a}{r^{3}}\right)|y|, \\
\mu(r, y)=\frac{a}{4 r^{3}} y^{2}+r_{c}\left(\frac{4}{3} \Lambda_{5}-\frac{a}{2 r^{3}}\right)|y| .
\end{gathered}
$$

Note that in these relations we have used $\Lambda_{5}=-\Lambda_{4}$ interchangeably. To find Schwarzschild solution in appropriate limit, we set $a=-2 m$, where $m$ is the Schwarzschild geometric mass. It is clear that in our linearized warped DGP setup, $\lambda(r, y=0) \neq-\nu(r, y=0)$ on the 3-brane. We expect that in the limit of $r_{c} \rightarrow \infty$ the solutions reduce to the 4D general relativistic solutions on the brane, that is, to a Schwarzschild- $(\mathrm{A}) \mathrm{d} \mathrm{S}_{(4)}$ metric. However, this has not occurred here. We note that the reason behind having a non-Schwarzschild$(\mathrm{A}) \mathrm{dS}_{(4)}$ solution can be explained through the fact that the curvatures of the bulk and brane spacetimes should not exactly cancel each other. In the next section we focus on this issue.

\section{Recovering the Schwarzschild solution on the brane}

To recover the Schwarzschild- $(\mathrm{A}) \mathrm{dS}_{(4)}$ solution on the brane, we note that all metric components should be much smaller than unity as a result of the weak field prescription. But this is violated by the linearized solutions we obtained in the last section. Nevertheless, if we look at the 5D bulk solutions Eqs. (40), (41) and (42), we will see that in 5D, $\lambda$ and $\mu$ are large for large $r_{c}$, ( see for instance [24], [23] and [9]). Although we are interested only in 4D solutions, the linearized theory is not applicable at large $r_{c}$. These features show that the completely linearized theory does not result in correct 4D Schwarzschild-(A)d $\mathrm{S}_{(4)}$ solutions (which are given by $\mu(r)=0, \lambda(r)=-\nu(r)$ and $\nu(r)=-\frac{2 m}{r}-\frac{1}{3} \Lambda_{4} r^{2}$ ). In fact, in the limit $r_{c} \rightarrow \infty$, but yet in the weak field regime, we cannot neglect all nonlinear terms in some subspaces. For instance, if $r_{c}^{2}|a| \gg r^{3}$, quantities such as $\lambda_{y}$ and $\mu_{y}$ are large enough that we have to save their squares in the field equations. Therefore, for $|a| \ll r \ll r_{*} \equiv\left(r_{c}^{2}|a|\right)^{\frac{1}{3}}$ the nonlinear field equations that should be solved are as follows ${ }^{\ddagger}$

$$
\begin{gathered}
\frac{2}{r}(\mu-\lambda)=-\nu_{r}-2 \mu_{r}+U(r), \\
4 r \mu_{r r}+8 \mu_{r}-4 \lambda_{r}-2 \nu_{r}+2 U(r)+2 r\left(\lambda_{y y}+2 \mu_{y y}\right)+r \lambda_{y}^{2}+3 r \mu_{y}^{2}+2 r \lambda_{y} \mu_{y}=4 r \Lambda_{5}, \\
2 \nu_{r}+2 U(r)+2 r \nu_{y y}+4 r \mu_{y y}+3 r \mu_{y}^{2}=4 r \Lambda_{5},
\end{gathered}
$$

${ }^{\ddagger}$ We note that a static source placed on a brane creates a nonzero scalar curvature around it. For a source of the size $<r_{*}$, this curvature extends to a distance $\sim r_{*}$. More intuitively, a static source distorts a brane medium around it creating a potential well, and the distortion extends to a distance $r \sim r_{*}$. This curvature suppresses nonlinear interactions that otherwise would become strong at the scale below $r_{*}$, see for instance $[14]$. 


$$
\begin{gathered}
2 r \nu_{r r}+2 r \mu_{r r}+2 \nu_{r}-2 \lambda_{r}+4 \mu_{r}+2 r\left(\nu_{y y}+\lambda_{y y}+\mu_{y y}\right)+r \lambda_{y} \mu_{y}+r\left(\lambda_{y}^{2}+\mu_{y}^{2}\right)=4 r \Lambda_{5}, \\
4 r \mu_{r r}+2 r \nu_{r r}+2 \nu_{r}-4 \lambda_{r}+8 \mu_{r}+2 U(r)+2 r \lambda_{y} \mu_{y}+r \mu_{y}^{2}=4 r \Lambda_{5},
\end{gathered}
$$

where $U(r)$ is an arbitrary function of $r$. Junction conditions in this situation are the same as the linearized case given by Eqs. (24), (25) and (26). By solving this set of equations, we find the following class of solutions

$$
\begin{gathered}
\nu(r, y)=\frac{a}{r}+\frac{1}{9} \Lambda_{5} r^{2}+\frac{4}{9} r_{c} \Lambda_{5}|y|+\frac{4}{27} r_{c}^{2} \Lambda_{5}^{2} y^{2}, \\
\lambda(r, y)=-\frac{a}{r}-\frac{1}{9} \Lambda_{5} r^{2}+\frac{4}{9} r_{c} \Lambda_{5}|y|, \\
\mu(r, y)=\frac{4}{9} r_{c} \Lambda_{5}|y|,
\end{gathered}
$$

where now $\Lambda_{4}=-\frac{1}{3} \Lambda_{5}$ with $\Lambda_{4}=-\frac{3}{4} \frac{1}{r_{c}^{2}}$. Note that in this framework we obtained a geometric interpretation of the brane cosmological constant in terms of the DGP crossover scale. Moreover, as we have mentioned at the end of the previous section, the curvatures of the bulk and brane spacetimes do not cancel each other exactly which is the reason behind having a non-Schwarzschild- $(\mathrm{A}) \mathrm{dS}_{(4)}$ solution in the previous section. On the brane with $y=0$, we find

$$
\begin{gathered}
\nu(r, y=0)=\frac{a}{r}-\frac{1}{3} \Lambda_{4} r^{2}, \\
\lambda(r, y=0)=-\frac{a}{r}+\frac{1}{3} \Lambda_{4} r^{2}, \\
\mu(r, y=0)=0,
\end{gathered}
$$

which is compatible with $4 \mathrm{D}$ Schwarzschild- $\mathrm{AdS}_{(4)}$ solution at the leading order if we set $a \equiv-2 m$

$$
d s^{2}=-\left(1-\frac{2 m}{r}-\frac{1}{3} \Lambda_{4} r^{2}\right) d t^{2}+\frac{d r^{2}}{\left(1-\frac{2 m}{r}-\frac{1}{3} \Lambda_{4} r^{2}\right)}+r^{2} d \Omega^{2} .
$$

Therefore, the Einstein gravity is recovered and the vDVZ problem is resolved by this strategy. Indeed, the spatial extrinsic curvatures of the brane, i.e. $\left.\lambda_{y}\right|_{y=0}$ and $\left.\mu_{y}\right|_{y=0}$, play a crucial role in the nonlinear nature of the solution and recovering the predictions of general relativity. In this respect, the nonlinear behavior arises from purely spatial geometric factors (see also Ref. [24]). We note also that there is a massless scalar mode, which arises from decomposition of five degrees of freedom of the bulk graviton in the massless limit (i.e. $\left.r_{c} \rightarrow \infty\right)$. This extra scalar mode persists as an extra degree of freedom in all regimes of the theory. The extrinsic curvature suppresses this extra scalar field inside the region $r \ll r_{*}$, whereas outside this region, the brane bending mode is free to propagate (see Ref. [9] for more details).

We note that Eqs. (48), (49) and (50) can be rewritten by using $\Lambda_{4}=-\frac{3}{4} \frac{1}{r_{c}^{2}}$ as follows 


$$
\begin{gathered}
\nu(r, y)=-\frac{2 m}{r}+\frac{1}{4} \frac{r^{2}}{r_{c}^{2}}+\frac{1}{r_{c}}|y|+\frac{3}{4} \frac{y^{2}}{r_{c}^{2}}, \\
\lambda(r, y)=\frac{2 m}{r}-\frac{1}{4} \frac{r^{2}}{r_{c}^{2}}+\frac{1}{r_{c}}|y|, \\
\mu(r, y)=\frac{1}{r_{c}}|y| .
\end{gathered}
$$

In the limit $r_{c} \rightarrow \infty$ we find the Schwarzschild solution on the brane. This means that the effect of the brane cosmological constant is negligible in this limit. Thus, it is essentially impossible to observe a Schwarzschild- $(\mathrm{A}) \mathrm{dS}_{(4)}$ interaction in our observations, and the Newtonian gravity is dominant at least in our solar system scale. In is important to note that the above solution is actually a black string solution since we find a singularity at $r=0$ for any value of $y$. This can be shown through singularity of the 5-dimensional Kretschmann scalar. The expression of the 5-dimensional Kretschmann scalar is to long to be presented here, but our calculation by using the Maple package, has shown that the 5-dimensional Kretschmann scalar is singular at $r=0$ for all values of $y$. In fact, in the $r \rightarrow 0$ limit, it reduces to the following simpler form

$$
\lim _{r \rightarrow 0} K^{2}=\frac{32 r_{c}^{3} y+16 r_{c}^{2} y^{2}+16 r_{c}^{4}}{\left(r_{c}+y\right)^{4}} \frac{1}{r^{4}}
$$

which obviously diverges at $r=0$. This shows that singularity at $r=0$ is an intrinsic singularity, and this is true for all values of $y$, which confirm that the solutions are actually black string. Moreover, the 4-dimensional Kretschmann scalar of the metric (54) expressed in terms of $r_{c}$ is given by

$$
K^{2} \equiv{ }^{(4)} R_{\alpha \beta \gamma \delta}{ }^{(4)} R^{\alpha \beta \gamma \delta}=\frac{3}{2} \frac{\left(\frac{r^{6}}{r_{c}^{4}}+32 m^{2}\right)}{r^{6}},
$$

which shows that singularity at $r=0$ on the brane is an intrinsic singularity. In the limit of $r_{c} \rightarrow \infty$, we have just one horizon which is the Schwarzschild horizon at $r=2 \mathrm{~m}$. But for $m=0$ there is no de Sitter horizon on the brane. The stability of black string solutions should be considered in the light of the results of Gregory-Laflamme pioneering work [27].

Another issue which requires especial attention here is the fact that a de Sitter bulk invalidates most of the attractive points of the Randall-Sundrum model such as the localization of the zero mode of the graviton near the brane. The question then arises: how is the situation in the warped DGP setup? In DGP-like models that have a 4D Ricci scalar in the bulk action, gravity becomes 5-dimensional at $r \gg r_{c}$ where 5D Einstein-Hilbert action is dominant. For $r \ll r_{c}$ gravity is 4-dimensional but not the 4D GR one. This property of these models is definitely different from RSII model, in which at low energies, $H \ell \ll 1,4 \mathrm{D}$ gravity is recovered to a good approximation. In RSII model what prevents gravity from leaking into the extra dimension at low energies is the negative bulk cosmological constant. Moreover, in RSII model the 4D gravitational constant strongly relies on the presence of the brane tension, and the brane tension should be positive to effective 4D gravitational constant have correct sign. This positive tension and the bulk negative cosmological constant are balanced with each other to have a zero 4D cosmological constant on the brane. 
In our warped DGP setup, we found $\Lambda_{4}<0$ and $\Lambda_{5}>0$, which is completely different from the RSII case. Here we don't need a negative $\Lambda_{5}$ and positive $\Lambda_{4}$ because induced gravity term gives us the desired results. Solutions that we have found in Sec 4, Eqs. (55)-(57), are valid only at $r \ll r_{*}$ and for $r \gg r_{c}$ appropriate solutions should be obtained. Nevertheless, since a warped DGP scenario is a hybrid braneworld model which contains both the pure DGP and RSII models in appropriate limits [6], it is expected that some of the mentioned shortcomings are still present in this case too. This needs further justification that we are going to study separately.

As the final remark, we note that Birkhoffs theorem is absent in theories of modified gravity such as the DGP braneworld scenario[28]. By calculation of the gravitational force on a test particle due to a spherical mass shell in the DGP setup, one can show that unlike in GR, the force depends on the mass distribution. In particular, the gravitational force within a spherical mass shell depends on the geometric structure of the bulk. In our setup, having Schwarzschild-(A)dS $\mathrm{d}_{(4)}$ solution means that Birkhoff's theorem holds good and this is due to geometric structure of the bulk manifold in this case which differs from pure DGP case.

\section{Summary}

In this paper we obtained a class of static black hole solutions in the warped DGP braneworld. Firstly we solved the general bulk field equations in the weak field limit (by linearizing the field equations and matching conditions). The solutions on the brane then were found by using the Israel matching conditions. However, these brane solutions were not compatible with Schwarzschild-(A) $\mathrm{dS}_{(4)}$ solutions on the brane. We adopted a strategy to solve this problem based on keeping appropriate nonlinear terms in the field equations. This strategy has its origin in the fact that the spatial extrinsic curvature of the brane plays a crucial role in the nonlinear nature of the solutions, and also in recovering the predictions of General Relativity. In fact, the nonlinear behavior arises from purely spatial geometric factors. Using this feature, we obtained some interesting black sting solutions compatible with well-known black hole solutions on the brane.

\section{References}

[1] C. Csaki, TASI Lectures on Extra Dimensions and Branes , [arXiv:hep-ph/0404096]. R. Maartens and K. Koyama,Brane-World Gravity, Living Rev. Relativity 13 (2010) 5, [arXiv:1004.3962].

[2] P. Horava and E. Witten, Hetrotic and Type I String Dynamics from Eleven Dimensions, Nucl. Phys. B 460 (1996) 506, [arXiv:hep-th/9510209].

[3] N. Arkani-Hamed, S. Dimopoulos and G. Dvali, The Hierarchy Problem and New Dimensions at a Millimeter, Phys. Lett. B 429 (1998) 263, [arXiv:hep-ph/9803315].

[4] L. Randall and R. Sundrum, A Large Mass Hierarchy from a Small Extra Dimension, Phys. Rev. Lett. 83 (1999) 3370, [arXiv:hep-ph/9905221].

L. Randall and R. Sundrum, An Alternative to Compactification, Phys. Rev. Lett. 83 (1999) 4690, [arXiv:hep-th/9906064]. 
[5] G. Dvali, G. Gabadadze and M. Porrati, 4D Gravity on a Brane in 5D Minkowski Space, Phys. Lett. B 485 (2000) 208, [arXiv:hep-th/0005016].

[6] K. -i. Maeda, S. Mizuno and T. Torii, Effective gravitational equations on a brane world with induced gravity, Phys. Rev. D 68 (2003) 024033, [arXiv:gr-qc/0303039].

[7] H. Collins and B. Holdom, Brane cosmologies without orbifolds, Phys. Rev. D 62, (2000) 105009.

Y. V. Shtanov, On Brane-World Cosmology, [arXiv:hep-th/0005193].

V. Sahni and Y. Shtanov, Braneworld models of dark energy, [arXiv:astro-ph/0202346].

[8] C. Deffayet, Cosmology on a brane in Minkowski bulk, Phys. Lett. B 502, (2001) 199, [arXiv:hep-th/0010186].

G. R. Dvali and G. Gabadadze, Gravity on a brane in infinite-volume extra space, Phys. Rev. D 63, (2001) 065007, [arXiv:hep-th/0008054].

C. Deffayet, G. R. Dvali and G. Gabadadze, Accelerated universe from gravity leaking to extra dimensions, Phys. Rev. D 65, (2002) 044023, [arXiv:astro-ph/0105068].

[9] A. Lue, The phenomenology of Dvali-Gabadadze-Porrati cosmologies, Phys. Rept. 423 (2006) 1-48, [arXiv:astro-ph/0510068].

[10] A. Chamblin, S. W. Hawking and H. S. Reall, Brane-World Black Holes, Phys. Rev. D 61 (2000) 065007, [arXiv:hep-th/9909205].

[11] N. Dadhich, R. Maartens, P. Papadopoulos and V. Rezania, Black holes on the brane, Phys. Lett. B 487 (2000) 1-6, [arXiv:hep-th/0003061].

[12] G. Kofinas, E. Papantonopoulos and V. Zamarias, Black Hole Solutions in Braneworlds with Induced Gravity, Phys. Rev. D 66 (2002) 104028, [arXiv:hep-th/0208207].

G. Kofinas, E. Papantonopoulos and V. Zamarias, Black Holes on the Brane with Induced Gravity, Astrophys. Space Sci. 283 (2003) 685, [arXiv:hep-th/0210006].

G. Kofinas, General Brane Dynamics with ${ }^{(4)} R$ term in the Bulk, JHEP 0108 (2001) 034, [arXiv:hep-th/0108013].

G. Kofinas, E. Papantonopoulos and I. Pappa, Spherically Symmetric Braneworld Solutions with ${ }^{(4)} R$ term in the Bulk, Phys. Rev. D 66 (2002) 104014, [arXiv:hep-th/0112019].

[13] R. Gregory, R. Whisker, K. Beckwith and C. Done, Observing braneworld black holes, JCAP 0410 (2004) 013, [arXiv:hep-th/0406252].

S. Creek, R. Gregory, P. Kanti and B. Mistry, Braneworld stars and black holes, Class. Quant. Grav. 23 (2006) 6633, [arXiv:hep-th/0606006].

[14] G. Gabadadze and A. Iglesias, Schwarzschild Solution In Brane Induced Gravity, Phys. Rev. D 72 (2005) 084024, [arXiv:hep-th/0407049].

[15] R. Gregory, Braneworld Black Holes, Lect. Notes Phys. 769 (2009) 259, [arXiv:0804.2595].

R. Whisker, Braneworld Black Holes, [arXiv:0810.1534]

[16] D. -C. Dai and D. Stojkovic, Analytic solution for a static black hole in RSII model, [arXiv:1004.3291] 
[17] C. Middleton and G. Siopsis, The Schwarzschild solution in the DGP model, Mod. Phys. Lett. A 19 (2004) 2259, [arXiv:hep-th/0311070].

E. Chang-Young and D. Lee, Charged Black Holes on DGP Brane, Phys. Lett. B 659 (2008) 58, [arXiv:0708.3032].

D. Lee, E. Chang-Young, M. Yoon, Charged Rotating Black Holes on DGP Brane, Int. J. Mod. Phys. A 24 (2009) 4389, [arXiv:0711.1074].

K. Zhang, $\mathrm{P}$. Wu and $\mathrm{H}$. $\mathrm{Yu}$, The stability of Einstein static universe in the DGP braneworld, Phys. Lett. B 690 (2010) 22, [arXiv:1005.4201].

[18] M. Heydari-Fard and H. R. Sepangi, Spherically symmetric solutions and gravitational collapse in brane-worlds, JCAP 02 (2009) 029, [arXiv:0903.0066]. M. Heydari-Fard, H. Razmi and H. R. Sepangi, Brane-World Black Hole Solutions via a Confining Potential, Phys. Rev. D 76 (2007) 066002, [arXiv:0707.3558].

M. Heydari-Fard, Black hole solutions in warped DGP brane world, Astrophys. Space Sci. 325 (2010) 287.

[19] Y. Iwasaki, Consistency Condition For Propagators, Phys. Rev. D 2 (1970) 2255.

H. van Dam and M. J. G. Veltman, Massive And Massless Yang-Mills And Gravitational Fields, Nucl. Phys. B 22 (1970) 397.

V. I. Zakharov, JETP Lett. 12 (1970) 312.

[20] A.I. Vainshtein, To The Problem Of Nonvanishing Gravitation Mass, Phys. Lett. 39B, (1972) 393.

[21] T. Damour, I. I. Kogan and A. Papazoglou, Spherically symmetric spacetimes in massive gravity, Phys. Rev. D 67, (2003) 064009, [arXiv:hep-th/0212155].

[22] A. Higuchi, Nucl. Phys. B282, (1987) 397; ibid. B325, (1989) 745.

I.I. Kogan, S. Mouslopoulos, and A. Papazoglou, The $m \rightarrow 0$ limit for massive graviton in $d S_{4}$ and $A d S_{4}$-How to circumvent the van Dam-Veltman-Zakharov discontinuity, Phys. Lett. B 503, (2001) 173, [arXiv:hep-th/0011138].

[23] C. Deffayet, G. R. Dvali, G. Gabadadze and A. I. Vainshtein, Nonperturbative Continuity in Graviton Mass versus Perturbative Discontinuity, Phys. Rev. D 65, (2002) 044026, [arXiv:hep-th/0106001].

A. Lue, Cosmic strings in a braneworld theory with metastable gravitons, Phys. Rev. D 66, (2002) 043509, [arXiv:hep-th/0111168].

M. Porrati, Fully covariant van Dam-Veltman-Zakharov discontinuity, and absence thereof, Phys. Lett. B 534, (2002) 209-215, [arXiv:hep-th/0203014].

[24] A. Gruzinov, On the Graviton Mass, New Astron. 10 (2005) 311, [arXiv:astro$\mathrm{ph} / 0112246]$.

[25] T. Tanaka, Weak gravity in DGP braneworld model, Phys. Rev. D 69, (2004) 024001, [arXiv:gr-qc/0305031].

[26] J. Garriga and T. Tanaka, Gravity in the Randall-Sundrum Brane World, Phys. Rev. Lett. 84, (2000) 2778, [arXiv:hep-th/9911055].

[27] Ruth Gregory, Raymond Laflamme, Evidence for the Stability of Extremal Black pBranes, Phys. Rev. D 51, (1995) 305, [arXiv:hep-th/9410050]. 
[28] A. Satz, F. D. Mazzitelli and E. Alvarez, Vacuum polarization around stars: Nonlocal approximation, Phys. Rev. D 71 (2005) 064001, [arXiv:gr-qc/0411046].

J. W. Moffat and V. T. Toth, Modified gravity and the origin of inertia, MNRAS (2009) 395, [arXiv:0710.3415].

D. -C. Dai, I. Maor and G. Starkman, Modified gravity: living without Birkhoff I. DGP, Phys. Rev. D 77 (2008) 064016 [arXiv:0709.4391]. 